\title{
Enhancement of Electron Movement in Cytochrome c protein with Water Hydration
}

\author{
Amba Datt Pant \\ Institute of Materials Structure Science, Muon Science Laboratory \\ High Energy Accelerator Research Organization (KEK), \\ 203-1 Shirakata, Tokai-mura, Naka-gun, Ibaraki 319-1106 Japan \\ Email:pant@post.kek.jp
}

\begin{abstract}
Muon spin rotation and relaxation $(\mu \mathrm{SR})$ measurements have been carried out on oxidized form of cytochrome c protein to understand the relation of electron transfer with water hydration at room temperature. Analysis of $\mu$ SR data following the Risch-Kehr theory shows that intra- and inter-chain electron movement in the protein enhanced significantly. The three-dimensional diffusion of electron in wet sample (20\% relative humidity) is increased around $75 \%$ with respect to that in dry sample ( $5 \%$ relative humidity).
\end{abstract}

Keywords: $\mu$ SR method, Electron transfer, Water hydration, Protein

\section{INTRODUCTION}

Electron transfer phenomenon plays important role in activity of animals and plants. For example, energy storage process in photosynthetic electron transfer chain and energy usage process in respiratory chain in mitochondria[1]. To understand the function of these biological systems, knowledge of electronmovement from one active redox site to another is necessary. During respiratory process electron transfer takes place through many protein complexes in inner membrane of mitochondria in which large amount of energy released for metabolism. Cytochrome c (hereafter cyt c) protein is one of the members of the electron transport chain in mitochondria membrane[2]. The electron transfer takes place through this protein during the next to final process of a cycle (Fig. 1). Furthermore, the study of electron transferhelps to understand the damage and repair of DNAand application to new bio-devices[3].

Positive muon $\left(\mathrm{m}^{+}\right)$which is like a light proton (mass $\mathrm{m}_{\mu} \approx 1 / 9$ $\mathrm{m}_{\mathrm{p}}$; magnetic moment $\mu_{\mathrm{m}}=3.18 \mu_{\mathrm{p}}$ ), acts as sensitive spin probe in materials. Its bound state with electron known asmuonium $(\mathrm{Mu})$ behaves as light isotope of hydrogen in materials. The positive muon probes the local electronic and spin states of surrounding atoms and molecules at the stopping site.Here, thespin relaxation of such muon based of magnetic interaction of muon and moving electron in materials has been observed. Details of $\mu$ SR technique can be available elsewhere [2].

The electron transfer has been successfully studied in biological macromolecules like polyacetylene[4], cytochrome c[5], myoglobin, cytochrome oxidase and DNA[6, 7]using muon labelled-electron method. The relaxation behavior of muon explains the movement of electron brought in by it. The dimensionality of electron motion depends on temperature, hydration and magnetic field as well. Temperature dependent nature of electron transfer indicates that the dimensionality of diffusion increases with temperature ofcyt $\mathrm{c}[8]$.

Theoretical work based on the first-principles calculation explains the possible stopping sites of muon in amino acids is electronegative groups ( $\mathrm{O}$ of unsaturated bond) [9] and $\mathrm{N}$ of pyrrole ring in heme group[10]. Furthermore, Scheicher et al [11] pointed out thestructural geometry and hyperfine interaction of muon and $\mathrm{Mu}$ in $\mathrm{A}$ and $\mathrm{B}$ form of DNA differs significantly. It has also been suggested that hydration water molecules accelerate electron transfer rate[12, 13]. When electron donor-acceptor distances are fairly long, the effect could not be ignored in the both cases of the electron transfer in proteins and nucleic acids.

Here the effect of addition of water hydration on electron activity in cyt c protein at room temperature is presented.

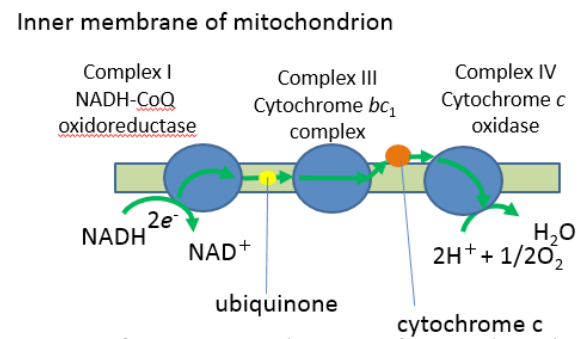

Fig. 1 Schematics of inner membrane of mitochondrion. Electron transfer takes place through cyt c protein. 


\section{METHOD}

According to muon labelled-electron method of $\mu \mathrm{SR}$ technique, during slowing down of implanted muon in soft materials like protein and DNA, it gets chance to pick up an electron and forms $\mathrm{Mu}$. This $\mathrm{Mu}$ stops in reactive sites of such macromolecules[2]. Consequently, the electron brought in by the muon shows characteristic behavior like localizes to form radicals or moves in the molecules which can be measured from the relaxation process of the muon. In the case of motion of the electron; one-, two- or threedimensional motion or diffusion or hopping of electron is possible. The behavior of the electron can be detected by measuring the spin relaxation of muon using $\mu \mathrm{SR}$ method. Cyt c sample was purchased from Sigma Aldrich Co LTD. Water content of the samples was adjusted around $5 \mathrm{wt} \%$ and $20 \mathrm{wt} \%$ in the case of dry (hereafter cyt c (dry)) and wet (hereafter cyt c (wet)) samples, respectively. A dry sample was prepared using dry nitrogen gas flow, and wetted samples with $\mathrm{H}_{2} \mathrm{O}$ using a wet gas generator (Shinei Co. LTD; model C-1). Water contents of the samples were analyzed by gravimetric measurements. The $\mu \mathrm{SR}$ measurements using pulsed muon at room temperature have been carried out in port 2, RIKEN-RAL, UK and D1-line, MLF, J-PARC, Japan. Based on characteristic properties of positive muon asymmetric decay and parity violation in weak interaction, decayed positronsfrom muons are collected by spectrometer around sample. Difference of forward and backward counts helps to calculate asymmetry at particular time as

Asymmetry $=\frac{\mathrm{FW}-\mathrm{BW}}{\mathrm{FW}+\mathrm{BW}} \times 100 \%$

Where FW is counts collected by forward counters and BW is that by backward counters. The time evolution of polarization (that is asymmetry) of an ensemble of muons implanted in a sample provides physical information about the interaction of the muon magnetic moment with its local environment.

In order to determine the relaxation rate of muon, RischKehr (RK) polarization function (Eq. 2 below) has been used.

\section{RESULT AND DISCUSSION}

The time evolution of asymmetry of cytc (dry) and cyt c (wet) at different magnetic fields from zero field (ZF) to longitudinal field (LF) $3950 \mathrm{G}$ are sown in Fig. 2 and Fig. 3 respectively. At ZF muon relaxation rate in cyt c (dry) is greater than that in cyt (wet). The data are fitted with RischKehr polarization function[14] except ZF. The ZF data are fitted using Lorentzian function.

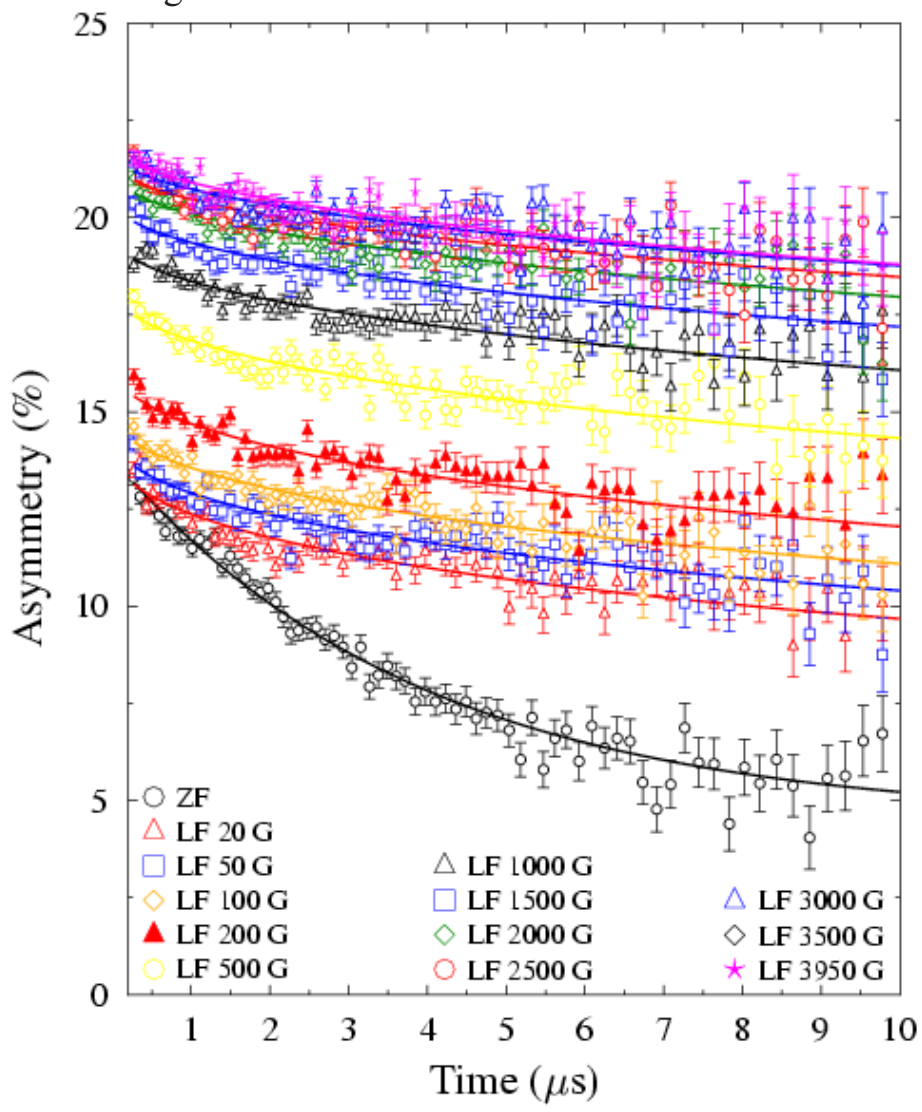

Fig. 2 Muon spin relaxation time spectra of cyt c (dry) at different fields from ZF to LF $3950 \mathrm{G}$. The solid lines are fitted lines of the corresponding data using $R K$ function except ZF data. The ZF data are fitted with Lorentzian function.

According to Risch-Kehr stochastic theory[14], polarization function of muon in terms of complementary error function is

$\mathrm{G}(\mathrm{t})=\exp (t) \operatorname{erfc}(t)^{1 / 2}$

Where RK relaxation parameter $=/\left(1+\left(2 \omega_{e}\right)^{\frac{1}{2}} \frac{D_{\|}}{\omega_{0}^{2}}\right)^{2}$

For the one-dimensional fast diffusion, $D_{\|>>}{ }^{\omega_{0}} w_{0}^{2} /\left(2 w_{e} l\right)^{1 / 2}$

$$
\begin{aligned}
& \mathrm{G}=w_{0}{ }^{4} / 2 w_{e} D_{\|}{ }^{2} \\
& D_{\|}=w_{0}{ }^{2} /\left(2 \gamma_{e} B \mathrm{G}\right)^{1 / 2} \\
& \Gamma \propto 1 / \mathrm{B}
\end{aligned}
$$

Where $\omega_{\mathrm{e}}$ is electronic Larmor frequency, $\omega_{0}$ is muon-electron hyperfine coupling, intra-chain diffusion rate and 1 electron spin flip rate.

In order to make further clear, let us take a schematic 
diagram as shown in Fig. 4[15, 16] where D represents inter-chain diffusion rate of electron. Inter-molecular and intra-molecular diffusion of electron can be considered as three-dimensional diffusion and one-dimensional transfer of electron in such macromolecules, respectively.

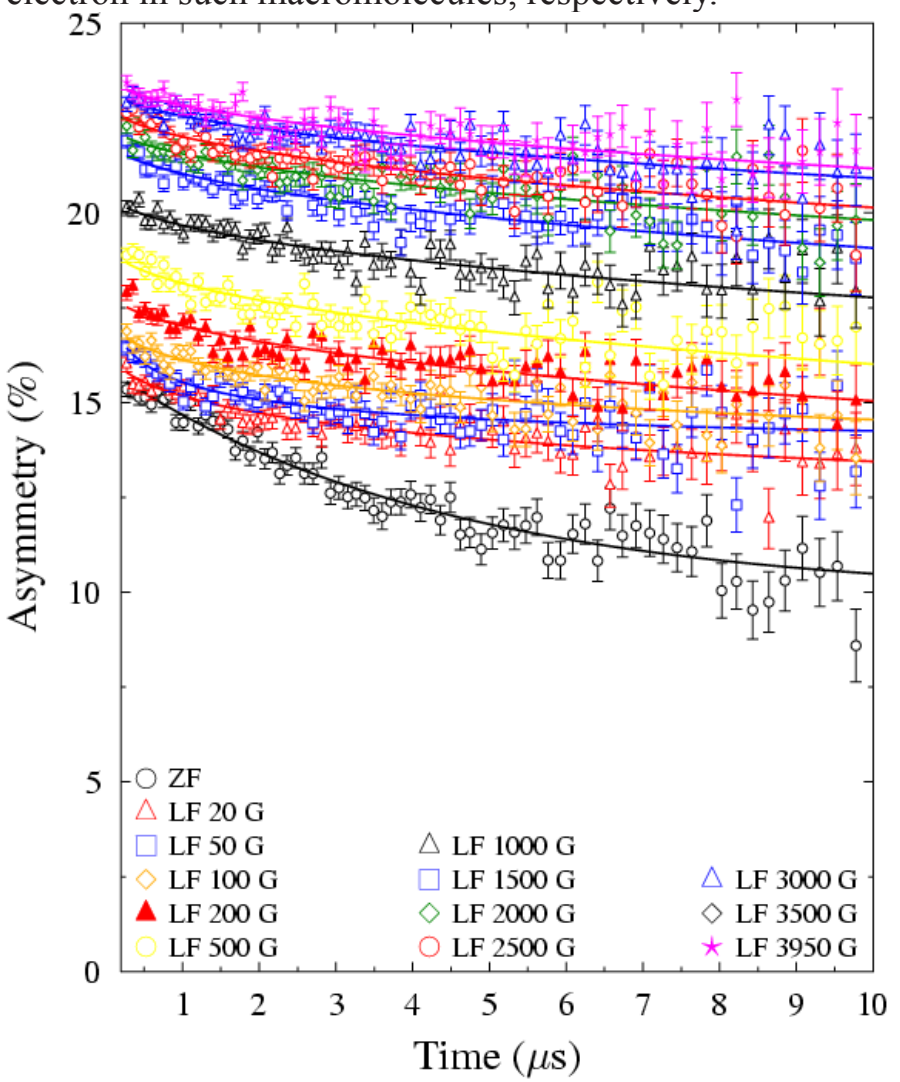

Fig. 3 Muon spin relaxation time spectra of cyt c (wet) at different fields from ZF to LF $3950 \mathrm{G}$. The solid lines are fitted lines of the corresponding data using $R K$ function except ZF data. The ZF data are fitted with Lorentzian function.

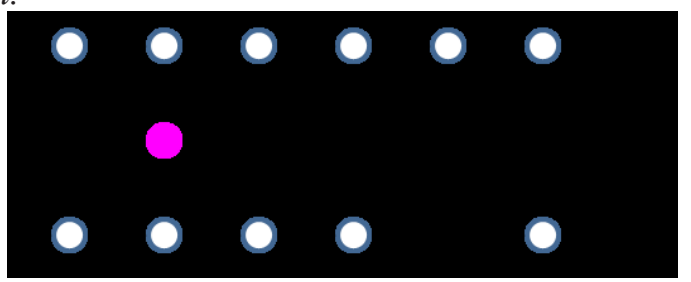

Fig. 4 Schematic of temporal model to show the intra- and interchain diffusion of electron in macromolecules [15].

One-dimensional fast diffusion of electron can explained based on inverse field dependent nature of RK relaxation parameter [Eq. 4]. In Fig. 5, athigher field where RK relaxation parameter is inverse of magnetic field is corresponds to intra-chain transfer of electron. However, RK theory is not sufficient to explain three-dimensional diffusion of electron. In addition, there is cut off field between such two different natures of the RK relaxation parameter. We can clearly see that cut off field $\left(B_{c}\right)$ is higher for wet sample than that of dry sample. Using temporal proposal by Pratt et al[15], the inter-chain diffusion rate $\left(D_{\wedge}\right)$ can be expressed as a function of cut off field ad, $\mathrm{D}_{\wedge}=\gamma_{\alpha} \mathrm{B}_{c}$ where $\gamma_{\mathrm{a}}$ is gyromatnetic ratio.

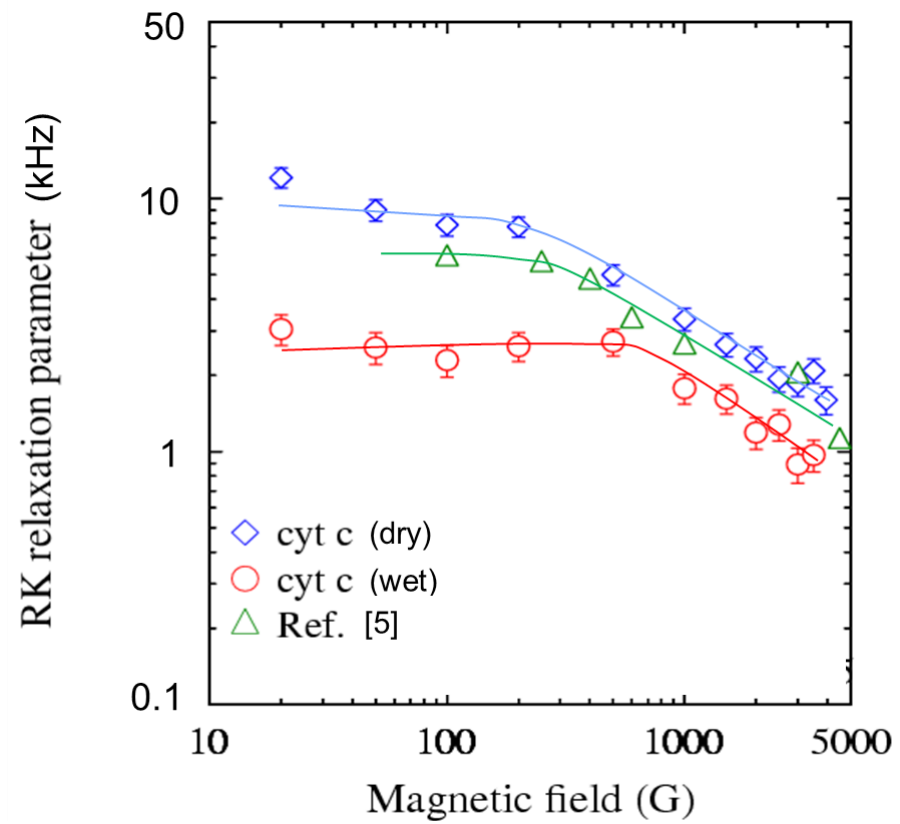

Fig. 5 Variation of RK relaxation parameter of cyt c (dry) and cyt $c$ (wet) with longitudinal magnetic field at room temperature.

From Fig. 5, the higher cut off field in cyt c (wet) represents that there is three-dimensional diffusion in wet sample. The ratio of intra-chain diffusion rate of electron in cyt c (wet) to that in cyt c (dry) as in Fig. 6 is around 1.3 which indicates the enhancement of one-dimensional diffusion with addition of water hydration. In the case of inter-chain diffusion which is corresponding to three-dimensional diffusion, the diffusion rate in cyt c (wet) is around $75 \%$ higher than that in cyt c (dry).

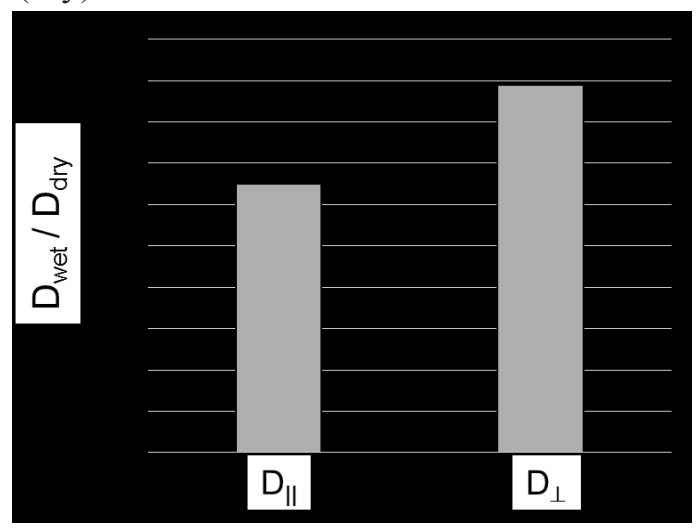

Fig. 6 Ratio of intra- and inter-chain diffusion rates of electron in cyt c (dry) and cyt c (wet). 


\section{CONCLUSION}

With the help of muon labelled-electron method, the observed electron diffusion rates in cyt $\mathrm{c}$ protein by adding water hydration up to $20 \%$ found significantly increased. The electron movement (transfer and diffusion) enhanced by adding water which is essential for activity of life. As a further work, the dynamic path of electron transfer in such macromolecules is to be studied. Furthermore, the use of reaccelerated micro-muon beam of ultra slow muon microscope will help to study such single crystal of proteins[17].

\section{ACKNOWLEDGEMENTS}

ADP would like to thank ProfessorsE. Torikai of University of Yamanashi; K. Nagamine of KEK, UCR Riverside; Y. Sugawara ofKitasato University; K. Shimomura of KEK, W. Higemotoof JAEA and F. Pratt of ISIS, RAL for their fruitful discussion and every support. This work was supported by Grant-in-Aid for Scientific Research on Innovative Areas of the Ministry of Education, Culture, Sports, Science and Technology (MEXT) Japan, Grant Number 23108003 on Frontier of Materials, Life and Elementary Particles Science explored by Ultra Slow Muon (FY2011-2015).

\section{REFERENCES}

1. Alberts, B., et al., Molecular biology of the cell. 2002(4th Ed.).

2. Nagamine, K., Introductory Muon Science. Introductory Muon Science, Cambridge University Press, 2007.

3. Torikai, E., et al., Electron transfer in DNA probed by the muon labelling method: A new interpretation. Physica B: Condensed Matter, 2006. 374-375: p. 441443.

4. Nagamine, K., et al., Solitons in Polyacetylene Produced and Probed by Positive Muons. Physical Review Letters, 1984. 53(18): p. 1763-1766.

5. K. Nagamine, et al., Intra- and inter-molecular electron transfer in cytochrome $c$ and myoglobin observed by the muon spin relaxation method. Physica B, 2000. 289: p. 631.
6. Torikai E., et al., Probing Electron Transfer in DNA New life science with muons. Hyperfine Interact., 2001. 138p.509.

7. Nagamine, K., et al., Molecular radiation biological effect in wet protein and DNA observed in the measurements of labeled electron with muons. Physica B: Condensed Matter, 2009. 404(5-7): p. 953-956.

8. A. D. Pant, et al., Hydration Effect on Electron Transfer in Cytochrome c Monitored by $\mu S R$. JPS Conf. Proc., 2015. 8: p. 033007-1.

9. D. Cammarere, et al., First-principle determination of muon and muonium trapping sites in horse heart cytochrome $c$ and investigation of magnetic hyperfine properties. Physica B, 2000. 289: p. 636-639.

10. R.H. Scheicher, et al., First-principles study of muon and muonium trapping in the protein chain of cytochrome c. Physica B, 2003. 326: p. 30-33.

11. Scheicher, R.H., et al., Comparative Theoretical Study of Hyperfine Interactions of Muonium in A- and B-Form DNA. Hyperfine Interactions, 2005. 158(1-4): p. 53-57.

12. Lin, J., I.A. Balabin, and D.N. Beratan, The nature of aqueous tunneling pathways between electron-transfer proteins. Science, 2005. 310(5752): p. 1311-1313.

13. Tomoyuki Hayashi and A.A. Stuchebrukhov, Electron tunneling in respiratory complex I. Proc. Natl. Acad. Sci., 2010. 107: p. 19157.

14. Risch, R. and K. Kehr, Direct stochastic theory of muon spin relaxation in a model for trans-polyacetylene. Physical Review B, 1992. 46(9): p. 5246-5257.

15. Pratt, F.L., Muon spin relaxation as a probe of electron motion in conducting polymers. Journal of Physics: Condensed Matter, 2004. 16: p. S4779.

16. Pratt, F.L., et al., Anisotropic polaron motion in polyaniline studied by muon spin relaxation. Physical Review Letters, 1997. 79(15): p. 2855-2858.

17. Nagamine, K., Past, present and future of ultra-slow muons. JPS Conf. Proc., 2014. 2: p. 010001. 